\title{
A 2-DOF Wideband Electrostatic Transducer for Energy Harvesting and Implantable Applications
}

\author{
Y. Zhu, S. O. R. Moheimani, M. R. Yuce \\ School of Electrical Engineering and Computer Science, the University of Newcastle \\ University Drive, NSW, 2308, Australia \\ Email: \{yong.zhu, mehmet.yuce, reza.moheimani\}@newcastle.edu.au
}

\begin{abstract}
This paper reports the modeling, fabrication and characterization of a SOI-based electro-mechanical transducer used for energy harvesting. The electrostatic transducer couples the ultrasonic energy with the vibration of a seismic mass, converting ultrasonic mechanical energy into electrical energy by means of polarized movable capacitor with comb structures. To extract ambient kinetic energy with arbitrary motion directions, this paper introduces a novel $2 \mathrm{D}$ resonator with resonance frequencies of $38520 \mathrm{~Hz}$ and $38725 \mathrm{~Hz}$. Working in diagonal direction, the $-10 \mathrm{~dB}$ bandwidth is $302 \mathrm{~Hz}$, twice wider than 1D resonator devices. The measured dynamic frequency response shows that a maximum peak-to-peak output voltage of $10 \mathrm{mV}$ can be obtained through a $1 \mathrm{M}$ ohm resistive load, and the harvested power is calculated as $0.1 \mathrm{nW}$. Potential applications for powering battery-free implanted biomedical devices are also discussed in this paper.
\end{abstract}

\section{INTRODUCTION}

A MEMS power generator can provide energy to low power autonomous microsystems from ambient energy sources such as vibration, acoustics, thermal and solar [1]. Kinetic energy is typically present in the form of vibration, random displacement, and is typically converted into electrical energy using electromagnetic, piezoelectric or electrostatic mechanisms [2-4]. However, the efficiency of vibrationelectricity conversion is quite low in existing micro generators, because: (a) most of the reported kinetic generator techniques suffer from scavenging in a narrow bandwidth from ambient sources due to their single resonance frequency; (b) most of them can only harvest energy in one single direction, which wastes the energy vibrating in other directions $[5,6]$.

A multifrequency piezoelectric converter was shown to broaden the bandwidth by using three piezoelectric bimorph cantilevers with identical dimensions but different masses [7]. Also, an $800 \mathrm{~Hz}$ wideband electromagnetic micro generator has been realized by implementing 40 serially connected cantilevers in different lengths [2]. Recently, U. Bartsch reported a two-dimensional electrostatic resonant micro energy generator to extract energy from ambient vibrations with arbitrary motion directions. However, the level of harvested power was too low (1-100 pW) [8].

This research is funded by Australian Research Council (ARC) discovery grant- DP0774287
In this paper, a novel 2-DOF (Degree-Of-Freedom) device topology is introduced, which can scavenge energy in arbitrary directions in a plane as well as broaden the bandwidth with two resonance frequency peaks. We also present an ultrasound-based method for powering battery-free implantable devices. Contrary to the RF methods, ultrasonic charging does not interfere with the electronic circuits of implanted devices and is relatively safe to a living tissue [9].

\section{DeVICE DESIGN}

To harvest vibration energy in arbitrary directions in a plane, a 2-DOF (Degree-Of-Freedom) motion mechanism has been incorporated in our design, as shown in Figure 1 (a). The seismic mass moves in $\mathrm{X}$ and $\mathrm{Y}$ axes to absorb vibration energy in any in-plane direction. This mechanism has mechanically decoupled $\mathrm{X}$ and $\mathrm{Y}$ modes, which makes it possible to detect the $\mathrm{X}$ and $\mathrm{Y}$ movements separately by using electrostatic combs. In Figure 1 (a), the $\mathrm{X}$ and $\mathrm{Y}$ mechanisms and the seismic mass are all discrete mechanical elements, attached to each other via elastic flexures. These flexures significantly decouple the $\mathrm{X}$ and $\mathrm{Y}$ movements of the mass.
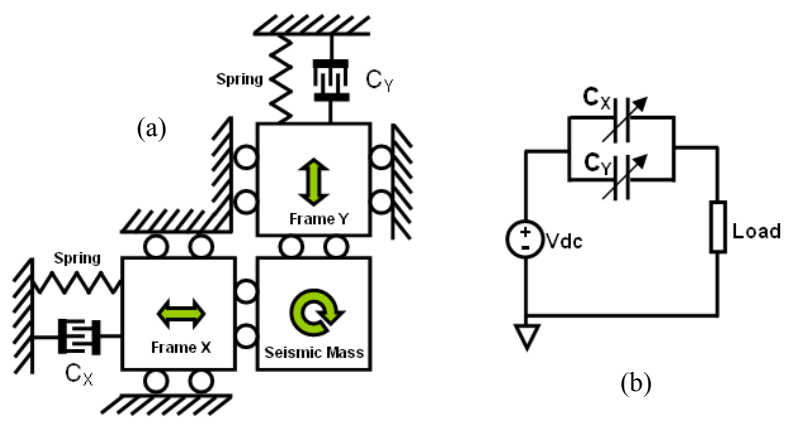

(b)

Fig. 1. 2-DOF motion mechanism to harvest any direction in-plane vibration energy. (a) schematic;(b) equivalent circuit model.

Figure 1 (b) shows the equivalent circuit model for the energy harvesting system. Electrostatic comb variable capacitors $C_{X}$ and $C_{Y}$ collect $X$ and $Y$ vibration contributions separately, and flow the currents into the load. The capacitors are biased with a $100 \mathrm{~V}$ DC voltage. If needed, this can be achieved using permanently charged electrets [10]. 


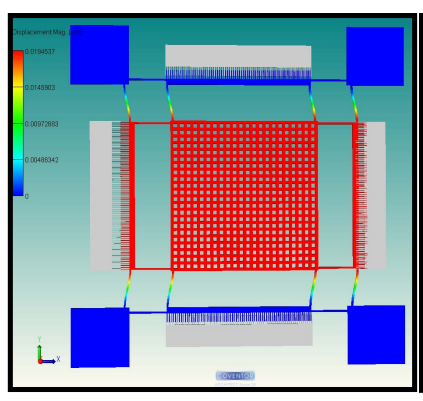

(a)

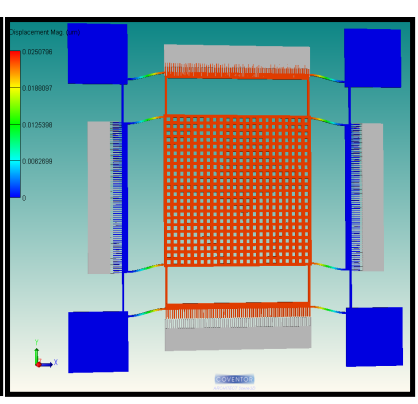

(b)
Fig. 2. Mechanical structure design showing two axis movement modes (a) and (b).

The detailed structures design is illustrated in Figure 2, which is inspired from a previous decoupled gyroscope structure [11]. The central seismic mass is suspended by 16 beams attached to four corner anchors. The specific topology of the beams splits a certain direction of seismic mass movement into the comb capacitance changing in $\mathrm{X}$ and $\mathrm{Y}$ frames. And the movable comb fingers are restricted by these beams and only move in parallel with fixed comb finger, without the risk of pulling in together. This way, symmetry of the structure is preserved, while the $\mathrm{X}$ and $\mathrm{Y}$ sense capacitors are kept independent from each other and contribute the electrical energy together.

For the structures in Figure 1 (a) and Figure 2, there are two primary resonance frequencies of $\mathrm{X}$ mode and $\mathrm{Y}$ mode. The resonance frequency is determined by the stiffness of the certain suspend beams and the mass in the movement direction. For instance, for $\mathrm{X}$ mode, the resonance frequency depends on the stiffness of the beams in parallel with $\mathrm{Y}$ axis, and the mass including the central mass and two movable $\mathrm{X}$ comb fingers. In our design, we keep all 16 beam dimensions identical, while changing the masses of $\mathrm{X}$ and $\mathrm{Y}$ movable comb fingers to adjust the resonance frequencies. To broaden the bandwidth, the masses were designed to have different dimensions along $\mathrm{X}$ and $\mathrm{Y}$ axes (the width of $\mathrm{X}$ frame is 1.5 $\mu \mathrm{m}$ wider than that of $\mathrm{Y}$ frame, as shown in Table I).

TABLE I.

DESIGN PARAMETERS FOR 2DOF ENERGY HARVESTERES

\begin{tabular}{cc}
\hline Parameter & Value \\
\hline Variable capacitor combs gap $(\mu \mathrm{m})$ & 2 \\
Beams length $(\mu \mathrm{m})$ & 255 \\
Beams width $(\mu \mathrm{m})$ & 12 \\
Seismic mass dimensions $\left(\mathrm{mm}^{*} \mathrm{~mm}\right)$ & $1^{*} 1$ \\
Structures thickness $(\mu \mathrm{m})$ & 25 \\
Width of X frame $(\mu \mathrm{m})$ & 40 \\
Width of Y frame $(\mu \mathrm{m})$ & 38.5 \\
$1^{\text {st }}$ resonance frequency $(\mathrm{kHz})$ & 39.238 \\
$2^{\text {nd }}$ resonance frequency $(\mathrm{kHz})$ & 39.266 \\
\hline
\end{tabular}

Table I shows the key design parameters for the energy harvester device. As the part of the design process, a modal analysis was carried out on the mechanical structure with a primary resonance frequency at $39238 \mathrm{~Hz}$ and the second resonance mode frequency at $39266 \mathrm{~Hz}$. The first two modes of the device obtained from a CoventorWare ${ }^{\mathrm{TM}}$ simulation are shown in Figure 2 (a) and (b). Simulation results indicate that in either mode, the central mass and combs only move in one single axis, showing good decoupling.

\section{FABRICATION}

The device was fabricated in a commercial Silicon-OnInsulator (SOI) MEMS foundry (MEMSCAP) process [12] with a $25 \mu \mathrm{m}$ thick device layer and minimum gap of $2 \mu \mathrm{m}$. Figure 3 provides an illustrated summary of this process:
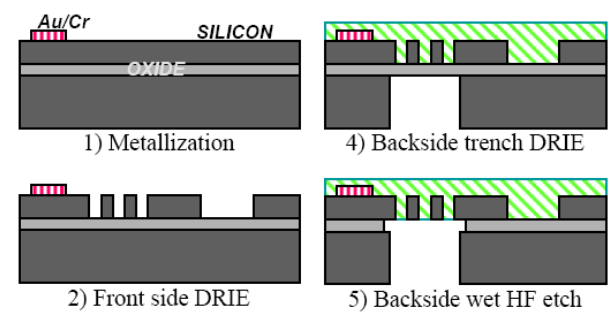

2) Front side DRIE
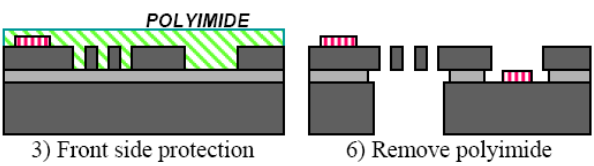

Fig. 3: SOI fabrication process through MEMSCAP

1) Surface metal pads are patterned on a highly doped ntype $25 \mu \mathrm{m}$ silicon device layer to allow for ohmic contact.

2) Deep reactive ion etch (DRIE) from the front side of the wafer to define both the anchored and movable features of the structure.

3) A protective polyimide layer is applied to the front side.

4) A deep trench underneath the movable structures is created by etching through the substrate using DRIE.

5) The exposed buried oxide is removed using a wet HF etch.

6) The polyimide coat on the front side is removed by oxygen plasma, thereby allowing the movable structure to be fully released. Then a large contact metal pad is patterned on the substrate for electrical contact.

The image of the whole device and a section of it taken under a Scanning Electron Microscope (SEM) are provided in Figure 4. There are a large number of holes in the central mass, making absorbing ultrasonic waves easier.

\section{EXPERIMENTAL RESULTS}

To validate the design in terms of energy harvesting, a proof-of-concept measurement has been performed using the fabricated device. The measurement set up is illustrated in Figure 5. The SUSS PM5 Analytical Probe System was adopted for the initial test. In Figure 5, the chip was fixed on the surface of an optical table, and then probes were placed on the device pads for electrical connection to provide access to the device for measurements.

An ultrasonic transducer (SQ-40T) was chosen to generate ultrasonic waves. This transducer has resonance frequency around $40 \mathrm{kHz}$, which conveniently close to the resonance 
frequencies of the designed $2 \mathrm{D}$ resonator. Thus, high energy coupling is expected to be achieved between the ultrasonic transducer and the power harvesting device. The ultrasonic transducer was driven by $4 \mathrm{~V}$ peak-to-peak ac output of Dynamic Signal Analyzer HP35670A to generate ultrasonic waves. The holes on the mass enhance the mechanical coupling between ultrasonic and seismic mass. The seismic mass absorbs this ultrasonic energy and vibrates in plane. The distance between the ultrasonic transducer and the fabricated device was $2 \mathrm{~cm}$. The smaller distance, the higher seismic mass vibration we may expect. While the mass is moving, the combs capacitance is changing also. As the comb capacitor is polarized by a dc voltage source, the moving combs force the currents into the input node of the signal analyzer (1M $\Omega$ input impedance). Sweeping the frequency of the drive voltage on the ultrasonic transducer, we can obtain the frequency response of the electrostatic energy harvester.

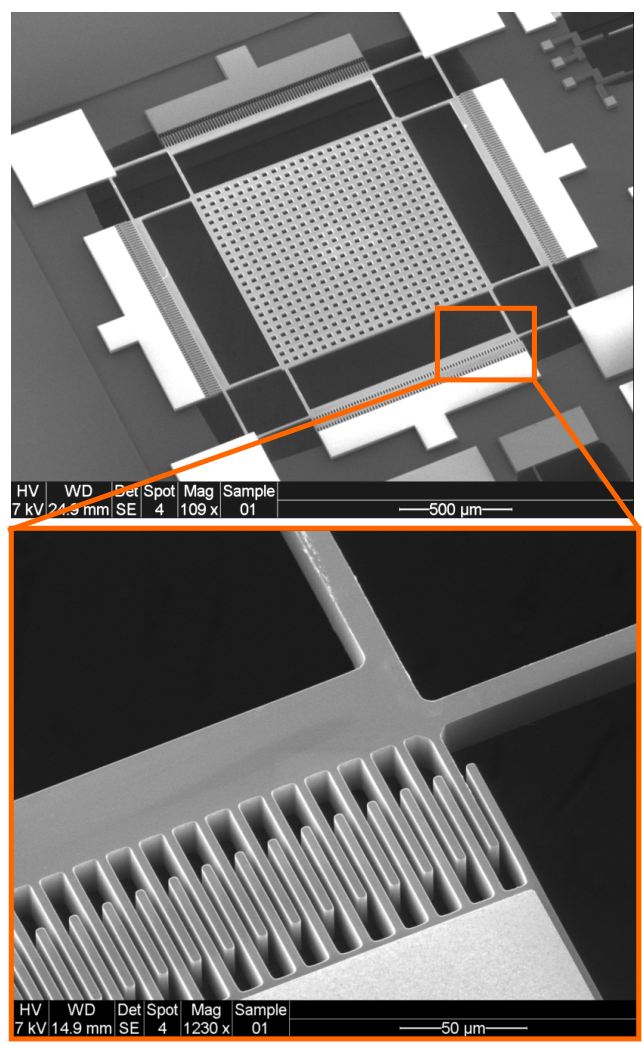

Fig. 4: SEM image of the 2-DOF electrostatic transducer.

Figure 6 shows the obtained magnitude response of the device, with the ultrasonic transducer aiming at diagonal direction, as shown in Figure 5. As the dynamics of the device in $\mathrm{X}$ and $\mathrm{Y}$ axes are different, there are two resonance frequency peaks in the frequency response of the system, located at $38520 \mathrm{~Hz}$ and $38725 \mathrm{~Hz}$. Note that the measured resonance frequencies are different with those predicted in the Table I. This is mainly due to the fabrication process, which is not free of errors. For instance, over etching in deep reactive ion etching (DRIE) makes the beams narrower that what was expected. Consequently, a lower stiffness in beams results in lower resonance frequencies.

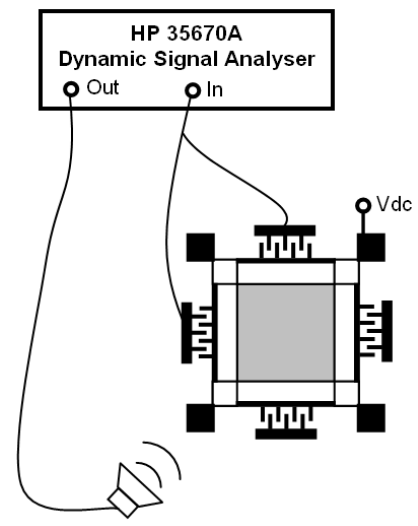

Fig. 5: Measurement setup.

The measured dynamic frequency response shows that a maximum peak-to-peak output voltage of $10 \mathrm{mV}$ was obtained through a $1 \mathrm{M} \Omega$ resistive load, and the harvested power is calculated to be $0.1 \mathrm{nW}$. As shown in Figure 6, the $-10 \mathrm{~dB}$ bandwidth is $302 \mathrm{~Hz}$, which is twice than a 1D resonator. However, the $10 \mathrm{mV}$ peak-to-peak output voltage is too low to trigger the rectifying diodes that need to be included for efficient electrical energy storage. In the test setup, two meter long cables attached to the probes, induced more than $400 \mathrm{pF}$ parasitic capacitance, which dramatically reduced the output voltage. A bonding wire instead of cables connected device is under testing, and is expected to result in a much higher output voltage.

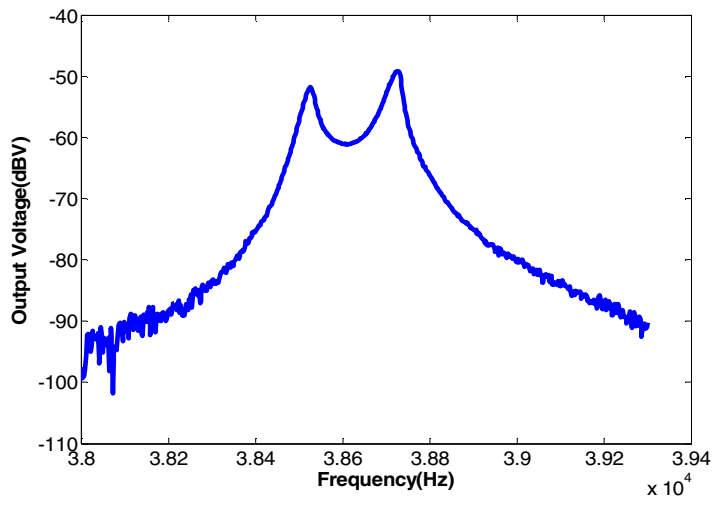

Fig. 6: Magnitude of the output voltage vs. frequency.

This work has a potential application in powering batteryfree implantable biomedical devices, by absorbing ultrasonic energy from outside of human body. Compared with radio frequency transmission methods [13], ultrasonic power transmission is relatively safe for human's health, and ultrasonic does not interfere with other electronic appliances. 


\section{CONCLUSION}

A 2-DOF electrostatic energy harvester was developed and characterized in this paper. The $2 \mathrm{D}$ resonator can extract ultrasonic energy from all directions in the device plane as well as broaden the bandwidth, increasing the efficiency of the energy scavenging. In the first prototype, $0.1 \mathrm{nW}$ power was harvested with a $10 \mathrm{mV}$ peak-to-peak voltage. However, the obtained $10 \mathrm{mV}$ peak-to-peak output is too low for the diodes rectification. This can be improved by wire bonding the connection directly to the diodes. Compared with radio frequency (RF) radiation methods, the ultrasonic power transmission is safe for the human's body and does not interfere with electronic devices and functions in medical environment.

\section{ACKNOWLEDGMENT}

The authors would like to thank Dave Phelan from the school of Medical Sciences at University of Newcastle for his help on SEM images.

\section{REFERENCES}

[1] S. P. Beeby, M. J. Tudor, and J. M. White, "Energy harvesting vibration sources for microsystems applications", Meas. Sci. Technol. 17 (2006), p175-195.

[2] I. Sari, T. Balkan, and H. Kulah, "An electromagnetic micro power generator for wideband envirnmental vibrations", Sensors and Actuators A, 145-146 (2008), p405-413.

[3] G. K. Ottman, H. F. Hofmann, A. C. Bhatt, and G. A. Lesieutre, "Adaptive piezoelectric energy havesting circuit for wireless remote power supply", IEEE Trans. on Power Electronics, vol.17, No.5, September 2002, p669-676.

[4] U. Bartsch, A. Trautmann, P. Ruther, J. Gaspar and O. Paul, "Electrostatic transducers for micro energy havesting based on SOI technology", Proc. of Transducers 2007, p141-144.

[5] M. Marzencki, Y. Ammar, and S. Basrour, "Integrated power harvesting system including a MEMS generator and a power management circuit", Sensors and Actuators A, 145-146(2008), p363370 .

[6] S. Kulkarni, E. Koukharenko, R. Torah, and J. Tudor, "Design, fabrication and test of integrated micro-scale vibration-based electromagnetic generator", Sensors and Actuators A , 145-146(2008), p336-342.

[7] M. Ferrari, V. Ferrari, M. Guizzetti, D. Marioli, and A. Taroni, "Piezoelectric multifrequency energy converter for power harvesting in autonomous microsystems", Sensors and Actuators A, 142 (2008), p329-335.

[8] U. Bartsch, J. Gaspar, and O. Paul, "A 2D electret-based resonant micro energy harvester", Tech. Digest IEEE MEMS 2009, January 2529, 2009, Sorrento, Italy, p1043-1046.

[9] S. Arra, J. Leskinen, J. Heikkila, and J. Vanhala, "Ultrasonic power and data link for wireless implantable applications", International Symposium on Wireless Pervasive Computing, p567-571, 2007.

[10] T. Sterken, P. Fiorini, G. Altena, C. Van Hoof, and R. Puers, "Havesting energy from vibrations by a micromachined electret generator", Proc. of Transducers 2007, p129-132.

[11] S. E. Alper, and T. Akin, "Symmetrical and decoupled nickel microgyroscope on insulation substrate", Sensors and Actuators A, 115(2004), p336-350.

[12] http://www.memscap.com/en_mumps.html

[13] http://www.minimitter.com/emitter.cfm 